\title{
Genome
}

\section{High temperature reduces peel color in eggplant (Solanum melongena) as revealed by RNA-seq analysis}

\begin{tabular}{|r|l|}
\hline Journal: & Genome \\
\hline Manuscript ID & gen-2019-0021.R2 \\
\hline Manuscript Type: & Note \\
\hline Author: & 06-Mate Submitted by the 2019 \\
\hline $\begin{aligned} \text { Complete List of Authors: } \\
\text { Keyword: }\end{aligned}$ & $\begin{array}{l}\text { Lv, Ling Ling; Chinese Academy of Tropical Agricultural Sciences, South } \\
\text { Feng, Xue Feng; Shangqiu University } \\
\text { Li, Wei; Chinese Academy of Tropical Agricultural Sciences, South } \\
\text { Subtropical Crops Research Institute } \\
\text { Li, Ke; Chinese Academy of Tropical Agricultural Sciences, South } \\
\text { Subtropical Crops Research Institute }\end{array}$ \\
\hline $\begin{array}{r}\text { Is the invited manuscript for } \\
\text { consideration in a Special } \\
\text { Issue? : }\end{array}$ & \begin{tabular}{l} 
Not applicable (regular submission) \\
\hline
\end{tabular} \\
\hline
\end{tabular}

\section{SCHOLARONE Manuscripts}




\section{High temperature reduces peel color in eggplant (Solanum}

\section{melongena) as revealed by RNA-seq analysis}

Ling Ling $\mathrm{Lv}^{1 *}$, Xue Feng Feng ${ }^{2}$, Wei $\mathrm{Li}^{1}, \mathrm{Ke} \mathrm{Li}^{1}$

1 South Subtropical Crops Research Institute, CATAS, Zhanjiang, Guangdong 524091, China / Hai-nan province key laboratory for postharvest physiology and technology of tropical hort icultural products, Zhanjiang, Guangdong 524091, China

2 Shangqiu University, Shangqiu, Henan 476000, China

* Correspondence: 15133253@qq.com

17

(8)

(1)

21




\section{Abstract}

To acquire the eggplant transcriptome under high temperature stress, eighteen cDNA libraries were constructed and sequenced. A total of $136.31 \mathrm{~Gb}$ clean data was obtained and $88.86 \sim 92.35 \%$ of the clean reads were mapped to the eggplant reference genome. Under high temperature, the number of down-regulated genes was more than that of up-regulated genes and there were more differentially expressed genes on the $10^{\text {th }}$ day after flowering than on the $15^{\text {th }}$ and $20^{\text {th }}$ days after flowering. On the $10^{\text {th }}$ day after flowering, the key genes $C H I, 3 G T, F 3^{\prime} 5^{\prime} H, D F R 2, A N S, F 3 H$ in anthocyanin synthetic pathway and most ERF, WRKY, bHLH and MYB transcription factors were all down-regulated. High temperature signifcantly decreased the total anthocyanin content in peels. The results showed that at the early stage of peel coloring, high temperature inhibited the expressions of key genes in anthocyanin biosynthetic pathways through the regulation of transcription factors, leading to a significant decrease in total anthocyanin content, which might reduce the peel color in eggplant.

Key words: eggplant, RNA-seq, peel coloring, anthocyanin, transcription factor, high temperature stress

\section{Introduction}

Eggplant (Solanum melongena L.) is a thermophile vegetable and is grown throughout China. However, consumers in different regions vary preference for the shape and color of eggplant. For example, green ovate eggplant is planted in Northeast China, purple eggplant in East China, green long eggplant in Central Plains, blackish purple eggplant in Southwest China and purplish red eggplant in South China. So the fruit color is one of the important factors in eggplant breeding. The optimum temperature for eggplant peel coloring is from 25 to $30{ }^{\circ} \mathrm{C}$, and the peels color will turn 
light even white when the temperature is over $35^{\circ} \mathrm{C}$. High temperature (HT) affects the appearance quality and reduces the commodity value of eggplant.

Flavonoids, including flavone, flavonol, isoflavone, flavanol, flavanone and anthocyanin, are a major class of secondary metabolites existed in all higher plants. They are very important pigments that contribute to a range of fruit colors from yellow to purple (Treutter 2005; Harborne and Williams 2000). Anthocyanins are one of the most abundant natural pigments and provide color to most flowers and fruits. Flavonoids, especially anthocyanins, are also thought to play important roles in protecting plant tissues from the damage of coldness and UV irradiation (Schulz et al. 2016; Takeda et al. 1994; Feild et al. 2001). The color of plant tissues is affected by intrinsic gene regulation and external environmental factors, such as light, temperature et al (Li et al. 2018; Ilk et al. 2015). Overexpression of MYB10.1/ $b H L H 3$ and MYB10.3/bHLH3 activated anthocyanin production by up-regulating NtCHS, NtDFR and NtUFGT, leading to pigmented peach fruit (Rahim et al. 2014).

The effects of HT on the anthocyanin content or the expression of genes in anthocyanin biosynthesis have been reported in some crops. In apple, HT caused a dramatic reduction of both peel anthocyanin concentration and transcripts of the genes in anthocyanin biosynthetic pathway (Lin-Wang et al. 2015). HT (maximum $35{ }^{\circ} \mathrm{C}$ ) reduced the total anthocyanin (TA) content to less than half of that in the control grape berries $\left(25{ }^{\circ} \mathrm{C}\right.$ ) (Mori et al. 2007). In rose flowers, transcript levels of CHS and dihydroflavonol reductase (DFR) decreased by $50 \%$ after $39^{\circ} \mathrm{C}$ heat treatment, suggesting that the decreased anthocyanin concentration is in part due to reduced transcription (Dela et al. 2003). However, no related research was reported in eggplant. The objective of this study is to understand which genes were up-regulated or down-regulated and which genes played key roles when HT stress caused the light peel color in eggplant. To understand the mechanism will be important for breeding new eggplant varieties with purplish red peel.

\section{Materials and methods}




\section{Plant materials}

Eggplant homozygous line E30 (Solanum melongena L.) with purplish red peel (Fig. 1A) was used as material. E30 that supports the findings of this study has been deposited in the CNGBdb (https://db.cngb.org/) with the accession code CNSebb2005576. When the plants had flower buds and did not flower, the six plants had been treated at $35{ }^{\circ} \mathrm{C} / 25{ }^{\circ} \mathrm{C}$ (light $12 \mathrm{~h} /$ dark $12 \mathrm{~h}$ ) and the other six plants used as control were treated at $25{ }^{\circ} \mathrm{C} / 20{ }^{\circ} \mathrm{C}$ (light $12 \mathrm{~h} /$ dark $12 \mathrm{~h}$ ) in the growth chambers respectively until the fruits were mature. On the $10^{\text {th }}$ day after flowering (10DAF), 15DAF and 20DAF, the peels were sampled and frozen in liquid nitrogen immediately, then stored at -80 ${ }^{\circ} \mathrm{C}$ until use. Each sampling point contained three independent biological replicates. When suffers HT stress, the peel color of E30 will turn light even white (Fig. 1B).

\section{RNA isolation, library construction and RNA sequencing}

Total RNA was isolated from peels using plant RNA extraction kit (TIANDZ, Inc Beijing, China, with DNase). The quantity and quality of the RNA samples were verified using Enzyme-linked immunosorbent analyzer (Spark $10 \mathrm{M}$, Switzerland) and agarose gel electrophoresis $(1.0 \%, w / v)$. Eighteen libraries were constructed and sequenced with PE150 using Illumina HiSeq 4000 platform by Genepioneer Biotech Corporation (Nanjing, China).

\section{Data processing and mapping}

Raw reads were cleaned by removing adaptor reads, ambiguous reads ('N' (represents A or T or $\mathrm{C}$ or $\mathrm{G}$ or $\mathrm{U}$ ) $>3 \%$ ), and low-quality reads (i.e., more than $50 \%$ of bases in a read had a quality value $(\mathrm{Q}$-score) $\leq 30)$ using perl script. High-quality reads were mapped to the eggplant reference genome (Hirakawa et al. 2014) on October 2017, using HISAT2 software (Kim et al. 2015).

\section{Functional annotation and classification}


All mapped genes and new genes found in this study were compared with public databases

including the Non-redundant nucleotide and protein database (Altschul et al. 1997), Swiss-Prot

database (Altschul and Gish 1996), the Clusters of Orthologous Groups of proteins database (Tatusov et al. 2003), Pfam database (Finn et al. 2016), using BLASTX, respectively, with an E-value of $1 \mathrm{e}^{-5}$. Then Web Gene Ontology Annotation Plot was used to obtain the GO functional classifications for all annotated genes (Ye et al. 2006). A Python script was used to retrieve Kyoto Encyclopedia of Genes and Genomes (KEGG) information from blast results and establish pathway associations between annotated genes and databases. Finally KEGG classification map was drawn.

\section{Differentially expressed genes (DEGs) analysis}

Fragments Per Kilobase of transcript per Million fragments mapped (FPKM) was applied to calculate and normalize the expression of the genes (Trapnell et al., 2010). The DESeq2 package (Love et al. 2014) was used to obtain the "base mean" value for identifying DEGs. Genes with False Discovery Rate $($ FDR $) \leq 0.05$ and the absolute value of $\log 2$ (Fold Change) $\geq 1$ were considered significant difference between the two libraries.

GO enrichment analyses of DEGs were performed using Singular Enrichment Analysis method with $P$-value $<0.01$ and FDR $<0.01$ by agriGO (Du et al. 2010). The hypergeometric Fisher exact test $(P$-value $<0.01)$ and Benjamini $($ FDR $<0.05)$ were performed to detect statistically significant enrichment of KEGG pathway. GO and KEGG enrichment analyses were performed using the whole eggplant genome as reference. Transcription factors (TFs) were identified using Plant TFDB 4.0 with default parameters (Jin et al. 2017). Heat maps of the gene sets were analyzed using R package 'pheatmap' (Kolde 2012). The gene expression levels were transformed to log2 (Fold Change) using three biological replicates.

\section{Real-time quantitative PCR (qRT-PCR) analysis}

The first-strand cDNA was synthetised from $2 \mu \mathrm{g}$ of total RNA using Prime Script RT Reagent Kit (Takara, Dalian, China). The qRT-PCR reactions were performed in 96-well plates using the ABI 
7500 fast Real-Time PCR system (Applied Biosystems, USA) with the SYBR Premix Ex Taq ${ }^{\mathrm{TM}} \mathrm{Kit}^{\mathrm{i}}$ (Takara, Dalian, China). The $18 S$ rRNA was used as an internal control for normalization of gene expression (Gantasala et al. 2013). The relative mRNA expression levels were calculated using the 2- $\Delta \Delta \mathrm{Ct}$ method (Livak and Schmittgen 2001). Three biological replicates and three technical repeats were performed for each gene and sample. Twenty microliters of the qRT-PCR reaction volume contained 10.0 $\mu$ l SYBR Premix Ex Taq ${ }^{\mathrm{TM}}$ II, $0.4 \mu \mathrm{l}$ ROX Reference Dye II (50×), $0.8 \mu \mathrm{l}$ PCR forward

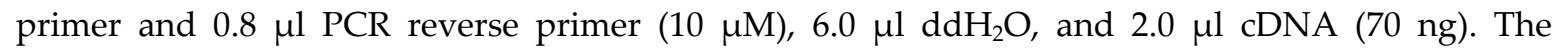
qRT-PCR parameters were: $95{ }^{\circ} \mathrm{C}$ for $5 \mathrm{~min}$, then 45 cycles of $95{ }^{\circ} \mathrm{C}$ for $10 \mathrm{~s}, 60{ }^{\circ} \mathrm{C}$ for $10 \mathrm{~s}$ and $72{ }^{\circ} \mathrm{C}$ for $10 \mathrm{~s}$. Melting curve and agarose gel electrophoresis analysis were performed to confirm the PCR specificity. The Minimum Information for publication of Quantitative real-time PCR Experiments (MIQE) was supplemented as Table S1.

The primers were designed using NCBI primer-BLAST (Ye et al. 2012). The information of genes and primer sequences were listed in supplemental Table S2.

\section{Extraction and analysis of total anthocyanin}

TAs were extracted from the peels according to the method described by Shao (2013) with minor modifications. In short, anthocyanins were extracted in $4 \mathrm{~mL}$ of acidic methanol $(2 \% \mathrm{HCl}$, $\mathrm{w} / \mathrm{v}$ ) by incubating $100 \mathrm{mg}$ of finely grounded peels at $32{ }^{\circ} \mathrm{C}$ for $2 \mathrm{~h}$ in dark, then clarified by centrifugation at $8,000 \times \mathrm{g}$ for $10 \mathrm{~min}$ at $4{ }^{\circ} \mathrm{C}$. The volume of the extract was made up to $10 \mathrm{~mL}$ with acidic methanol $(2 \% \mathrm{HCl}, \mathrm{w} / \mathrm{v})$. The content of TA was quantified based on the absorption of the extracts using the equation:

The content of TA $(\mathrm{mg} / \mathrm{g})=\left(\mathrm{A}_{530}-0.25 \times \mathrm{A}_{700}\right) \times \mathrm{FW}^{-1}$

Where $A_{530}$ and $A_{700}$ are the absorptions at $530 \mathrm{~nm}$ and $700 \mathrm{~nm}$ respectively; $\mathrm{FW}$ is the fresh weight of plant materials (g). Anthocyanins were quantified as triplicates of three independent biological replicates

\section{Statistical analysis}



were estimated with the Duncan test $(p<0.01)$.

157 (1)

\section{Results}

\section{RNA-seq data output, quality assessment and mapping}

In this study, eighteen cDNA libraries were constructed and sequenced on Illumina HiSeq 4000. The detailed information of the RNA-seq data was listed in Table 1. The rawdata was submitted to NCBI and the BioProject ID is PRJNA531285. A total of $136.31 \mathrm{~Gb}$ clean data was obtained. On average, 7.57 Gb clean data was obtained from each sample. The percentages of Phred-like quality score at the Q30 level (an error probability of $1 \%$ ) ranged from $88.24 \%$ to $93.83 \%$ and the average GC content was $42.54 \%$. Among the 18 samples, $88.86 \%$ to $92.35 \%$ of the clean reads were mapped to the eggplant reference genome and about $69.13 \%$ of the mapped reads were located at the exon region. The RNA-seq data showed that the sequencing quality was high and sequencing depth was sufficient for transcriptome coverage.

(1)
(1) (1) (15 6

(1)


Table 1 Summary of the sequencing data of 18 eggplant cDNA libraries

\begin{tabular}{|c|c|c|c|c|c|c|c|}
\hline \multirow{3}{*}{ Samples } & \multirow{3}{*}{ ID } & \multicolumn{2}{|l|}{ Reads } & \multicolumn{2}{|l|}{ GC } & \multirow{3}{*}{ Reads aligned } & \multirow{3}{*}{ Exonic } \\
\hline & & & Base number & & $\% \geq Q 30$ & & \\
\hline & & number (PE) & & content & & & \\
\hline s25-10-1 & T01 & 28405481 & 8521644300 & 42.90 & 93.70 & $52051438(91.62 \%)$ & $34,695,222(70.03 \%)$ \\
\hline s25-10-2 & T02 & 31048198 & 9314459400 & 42.71 & 93.11 & $56876777(91.59 \%)$ & $37,571,420(69.33 \%)$ \\
\hline s25-10-3 & T03 & 28121326 & 8436397800 & 42.82 & 93.31 & $51404232(91.40 \%)$ & $33,619,685(68.55 \%)$ \\
\hline s25-15-1 & T04 & 22032970 & 6609891000 & 42.60 & 91.66 & $40194817(91.22 \%)$ & $26,700,374(69.71 \%)$ \\
\hline s25-15-2 & T05 & 24308360 & 7292508000 & 42.75 & 93.80 & $44574698(91.69 \%)$ & $29,778,364(70.13 \%)$ \\
\hline s25-15-3 & T06 & 25843906 & 7753171800 & 42.59 & 88.24 & $46720964(90.39 \%)$ & $30,647,828(68.76 \%)$ \\
\hline s25-20-1 & T07 & 23646322 & 7093896600 & 42.40 & 90.90 & $43109128(91.15 \%)$ & $28,224,594(68.75 \%)$ \\
\hline s25-20-2 & T08 & 25182444 & 7554733200 & 42.60 & 91.54 & $44754546(88.86 \%)$ & $29,603,628(69.55 \%)$ \\
\hline s25-20-3 & T09 & 24578380 & 7373514000 & 42.71 & 91.58 & $44830771(91.20 \%)$ & $29,966,142(70.29 \%)$ \\
\hline s35-10-1 & $\mathrm{T} 10$ & 26158670 & 7847601000 & 42.32 & 90.61 & $47817035(91.40 \%)$ & $30,726,052(67.31 \%)$ \\
\hline s35-10-2 & $\mathrm{T} 11$ & 25959333 & 7787799900 & 42.45 & 91.26 & $47944959(92.35 \%)$ & $31,340,645(68.56 \%)$ \\
\hline s35-10-3 & $\mathrm{T} 12$ & 23620728 & 7086218400 & 41.96 & 91.01 & $43078441(91.19 \%)$ & $26,612,496(64.71 \%)$ \\
\hline s35-15-1 & $\mathrm{T} 13$ & 25163461 & 7549038300 & 42.36 & 90.42 & $45896870(91.20 \%)$ & $30,110,034(68.81 \%)$ \\
\hline s35-15-2 & $\mathrm{T} 14$ & 23181129 & 6954338700 & 42.38 & 90.74 & $42322650(91.29 \%)$ & $28,131,775(69.67 \%)$ \\
\hline s35-15-3 & $\mathrm{T} 15$ & 24558310 & 7367493000 & 42.17 & 90.24 & $44712476(91.03 \%)$ & $29,396,120(68.91 \%)$ \\
\hline s35-20-1 & $\mathrm{T} 16$ & 21676922 & 6503076600 & 42.57 & 91.04 & $39381141(90.84 \%)$ & $26,283,534(70.10 \%)$ \\
\hline s35-20-2 & $\mathrm{T} 17$ & 25364209 & 7609262700 & 42.64 & 93.77 & $46484804(91.63 \%)$ & $31,289,063(70.68 \%)$ \\
\hline s35-20-3 & $\mathrm{T} 18$ & 25504099 & 7651229700 & 42.75 & 93.83 & $46754110(91.66 \%)$ & $31,385,277(70.54 \%)$ \\
\hline average & & 25241902.67 & 7572570800 & 42.54 & 91.71 & $46050548(91.21 \%)$ & $30337903(69.13 \%)$ \\
\hline total & & 454354248 & & & & & \\
\hline
\end{tabular}




\section{Analysis of total anthocyanin}

At different stages of fruit development and different temperatures, the changes of TA content

were showed in Fig. 1C. At $25{ }^{\circ} \mathrm{C}$ (Control), the TA content in peels increased gradually with the

fruit development. Compared to control, the TA content signifcantly decreased at all three time points under HT stress. The results showed that HT signifcantly decreased the TA content in peels.

\section{Identification of DEGs}

A total of 9,065, 5,845 and 6,629 genes were differentially expressed under $35{ }^{\circ} \mathrm{C}$ stress compared to $25{ }^{\circ} \mathrm{C}$ on 10DAF, 15DAF and 20DAF respectively. The number of up-regulated genes was less than that of down-regulated genes, which suggested HT might suppress the expression of many peel coloring related genes. There were more DEGs on 10DAF than 15DAF and 20DAF, which implied that HT had a severe effect at the early stage of peel coloring. As shown in Fig. 2, under HT stress, there were 497 co-up-regulated genes and 1,328 co-down-regulated genes at three time points.

\section{Gene ontology (GO) classification and enrichment analysis of DEGs}

Under HT stress, 5,086, 3,267 and 4,036 DEGs were significantly enriched on 10DAF, 15DAF and 20DAF respectively, which were classified into 76, 47, and 117 GO terms respectively (Fig. S1A-C). On 10DAF and 15DAF, the DEGs related to peel coloring were presented in the biological process, and in Anthocyanin-containing compound biosynthetic process (GO: 0009718, Fig. S1A-B), sixteen and fourteen DEGs were enriched respectively. Among the annotated DEGs, only one gene (Sme2.5_00096.1_g00007.1) was up-regulated, while the other DEGs were all down-regulated. The co-down-regulated DEGs were focused on anthocyanidin-3-O-glucosyltransferase-like (3GT, 
Sme2.5_06210.1_g00004.1), flavonoid 3',5'-hydroxylase $\quad\left(F 3^{\prime} 5^{\prime} H, \quad\right.$ Sme2.5_04313.1_g00001.1, XLOC_046562, XLOC_046563), DFR2 (Sme2.5_01401.1_g00004.1) and anthocyanidin synthase (ANS, Sme2.5_01638.1_g00003.1, Sme2.5_01638.1_g00005.1, XLOC_025201).

On 20DAF, most of the terms in biological process were closely related to defense, such as Response to blue light (GO: 0009637) and Defense response to bacterium (GO: 0042742). Anthocyanin-containing compound biosynthetic process (GO: 0009718) and Flavonoid biosynthetic process (GO: 0009813) were also enriched in GO classification but not showed in Fig. S1C.

\section{KEGG Pathway Classification and Enrichment Analysis of DEGs}

All DEGs were BLAST against the KEGG Ontology database. On 10DAF, 1,233 DEGs were classified into 13 KEGG categories. The top 8 categories were presented in Fig. 3A and there were 20 DEGs in Flavonoids biosynthesis (ko00941). On 15DAF and 20DAF, there were 12 and 14 DEGs in Flavonoids biosynthesis respectively (Fig. 3B-C).

The Flavonoid biosynthesis category was found at all three time points (Fig. 3) and 22 DEGs were involved (Fig. 4). Caffeoyl-CoA O-methyltransferase (CCoAOMT) and Cytochrome P450 (CYP) were all down-regulated by HT stress. CYP 98A3-2, F3H and CHI were down-regulated just on 10DAF. The key genes CHS (Sme2.5_01077.1_g00016.1, Sme2.5_13923.1_g00001.1), DFR2, ANS2, ANS, F3'5'H and F3'H were down-regulated on 10DAF and/or 15DAF, but up-regulated on 20DAF.

\section{Validation of RNA-seq data by qRT-PCR analysis}

To validate the expression data of RNA-seq, twelve DEGs were selected for qRT-PCR analysis (Fig. 5), including two TFs (SmMYB12 and SmWD40), six genes involved in anthocyanin biosynthesis (SmDFR2, SmANS2, SmANS, SmF3'5'H, SmCHS and SmCHS2), one regulating anthocyanin metabolism (purple acid phosphatase 1, SmPAP1), one in lignin biosynthesis (SmCCoAOMT-3), one in isoflavone biosynthesis (SmCYP-2) and one flavonol synthase (SmFLS). To compare the expression data between RNA-seq and qRT-PCR, the relative expression level was 
transformed to log2 (Fold Change). As Fig. 5 showed, the expressions of SmF3'5'H1, SmCHS, and

SmMYB12 were higher in RNA-seq than in qRT-PCR. In contrast, the expressions of SmCCoAOMT-3,

SmCYP-2 and SmWD40 were lower in RNA-seq than in qRT-PCR. Though the expression trends of these genes were different, the qRT-PCR results showed a high consistency with RNA-seq data which suggested that RNA-Seq data was credible and could be used for subsequent experiments.

\section{DEGs related to transcription factors}

Under HT stress, comparing with control, a total of 205, 131 and 154 TFs were differentially expressed on 10DAF, 15DAF and 20DAF respectively (Fig. 6). Among the differentially expressed TFs, the top 5 of ERF, WRKY, bHLH, MYB and NAC families were enriched at all three time points.

\section{Discussion}

The peel color is an important economic trait for eggplant and is essentially determined by the components and content of anthocyanins which are modulated by the genes in flavonoid biosynthetic pathway or the TFs. Structural genes SmF3'5'H, SmDFR, Sm3GT and Sm5GT were highly expressed in peels of purple eggplant (Wang 2016). SmCHS, SmCHI, SmDFR, SmF3'5'H and SmANS had the highest expression in purple peels which showed correlation with anthocyanin content (Jiang et al. 2016). Stommel (2015) reported that anthocyanin content increased 2003). significantly in developing purple eggplant fruit, levels nearly 500-fold greater than those measured in white fruit. Coincident with anthocyanin content, the transcript levels of biosynthetic genes $\mathrm{CHS}$, DFR, ANS were significantly higher in violet tissue than in white tissue (Stommel and Dumm 2015). Transcript levels of CHS and DFR decreased by $50 \%$ after heat treatment, suggesting that the decreased anthocyanin concentration in rose flower is in part due to reduced transcription (Gal et al.

In this study, on 10DAF and 15DAF, in Flavonoid biosynthetic category, only one annotated DEG (CHS, Sme2.5_00283.1_g00002.1) was up-regulated, and the other DEGs were all 
down-regulated under HT stress (Fig. 4). Most of the down-regulated DEGs (other CHS homologs,

CHI, F3H, DFR2, ANS, F3' $5^{\prime} H$ and $F 3^{\prime} H$ ) are the key genes in anthocyanin synthetic pathway (Fig. 7). CYPs and CCOAOMTs were the key genes in lignin biosynthetic pathway (Fig. 7) which played important roles in abiotic stresses (Lin et al. 2012). Winkel-Shirley (2001) reported that methylation mediated by CCoAOMT could improve the stability of anthocyanin. In this study, CCoAOMTs were down-regulated by HT stress, which was unfavourable for anthocyanin accumulation. Under HT stress, the TA content signifcantly decreased in eggplant peels at all three time points (Fig. 1C). Pavel (2018) reported that the color of grain was determined mainly by anthocyanin content and composition. The above results indicated that HT inhibited the expressions of anthocyanin synthesis related genes and decreased the TA content, which might lead to the light peel color in eggplant. The results are agreement with previous studies (Lin-Wang et al. 2015; Mori et al. 2007; Wang 2016; Jiang et al. 2016; Stommel and Dumm 2015; Gal et al. 2003; Rowen et al. 2009; Poudel et al. 2009). On 20DAF, the key genes in anthocyanin biosynthesis, such as CHS, DFR2, ANS, ANS2 and F3'H were up-regulated (Fig. 4), resulting in anthocyanins accumulation, which might protect the eggplant fruits from the damage of HT. The similar phenomenon was reported in Arabidopsis that most flavonols and anthocyanins accumulated upon cold exposure (Schulz et al. 2016).

TFs have already been reported to play important roles not only in abiotic stresses but also in flavonoids or anthocyanin biosynthesis. The expressions of SmMYB1, SmMYB2 and SmAN11 in purple eggplant were higher than those in white eggplant; Overexpression of SmMYB1 promoted anthocyanin accumulation in the regenerating shoots of eggplant (Zhang et al. 2014). Overexpression of VvbHLH1 significantly increased the accumulation of flavonoids and enhanced salt and drought tolerance in transgenic Arabidopsis thaliana (Yang et al. 2016). PbMYB10b regulated the anthocyanin and proanthocyanidin pathways by inducing the expression of $P b D F R$; PbMYB9 induced the synthesis of anthocyanins and flavonols in pear fruits (Zhai et al. 2016). VvWRKY26 is involved in flavonoid biosynthesis (Amato et al. 2016). The heterodimer of a NAC TF and PpNAC1 
can activate the transcription of $P p M Y B 10.1$, resulting in anthocyanin pigmentation in tobacco (Zhou et al. 2015).

In this study, under HT stress, ERF, WRKY, bHLH, MYB and NAC were differentially expressed compared with control (Fig. 6). All the differentially expressed ERFs, WRKYs, MYBs and bHLHs were showed in Figure S2-S5 respectively and the number of down-regulated TFs was more than that of up-regulated, especially on 10DAF. There were four TFs (WIN1-2, WRKY19-2, MYB12 and MYB113) up-regulated at three time points, which might negatively regulate the peel coloring. HT significantly down-regulated the expression of ERF1B-1, ERF4, WRKY45, WRKY31-2, MYB39, MYB108, bHLH14 and NAC1, which might postively regulate the peel coloring. In a word, differentially expressed ERF, WRKY, NAC, MYB and bHLH might play important roles in peel coloring, especially at the early stage of fruit development.

The results indicated that on the one hand HT stress inhibited the expressions of anthocyanin synthesis related genes (Fig. 4) through the regulation of TFs, on the other hand, HT signifcantly decreased the TA content in peels (Fig. 1C), which might lead to the light peel color in eggplant.

\section{Acknowledgements}

This work was funded by the Project of Natural Science Foundation of Guangdong Province (2015A030310452), the Fundamental Research Funds for South Subtropical Crops Research Institute , CATAS (1630062017030). The authors are grateful to Xiao Xi Ou, Li Ming Zhi and Gao Xiao Min for their technical assistance.

Conflict of Interest: The authors declare no conflict of interest.

\section{References}

Altschul, S. F., and Gish, W. 1996. Local alignment statistics. Method Enzymol. 266:460-480. doi: 10.1016/S0076-6879(96)66029-7 
Altschul, S. F., Madden, T. L., Schaeffer, A. A., Zhang, J., Zhang, Z., Miller, W., Lipman, D.J. 1997. Gapped BLAST and PSI-BLAST: a new generation of protein database search programs. Nuleic Acids Res. 25: 3389-3402. doi: 10.1093/nar/25.17.3389

Amato, A., Cavallini, E., Zenoni, S., Finezzo, L., Begheldo, M., Ruperti, B., Tornielli, G.B. 2016. A grapevine TTG2-like WRKY transcription factor is involved in regulating vacuolar transport and flavonoid biosynthesis. Front. Plant Sci. 7:1979. doi:10.3389/ fpls.2016.01979

Dela, G., Or, E., Ovadia, R., Nissim-Levi, A., Weiss, D. \& Oren-Shamir, M. 2003. Changes in anthocyanin concentration and composition in 'jaguar' rose flowers due to transient high-temperature conditions. Plant Sci. 164(3):333-340.

Du, Z., Zhou, X., Ling, Y., Zhang, Z., Su, Z. 2010. agriGO: a GO analysis toolkit for the agricultural community. Nucleic Acids Res. 38 (suppl_2):W64-W70. doi: 10.1093/nar/gkq310

Feild, T. S. , Lee, D. W., Holbrook,N. M. 2001. Why leaves turn red in autumn. the role of anthocyanins in senescing leaves of Red-osier Dogwood. Plant Physiology. 127(2):566-574. doi: https://doi.org/10.1104/ pp.010063

Finn, R. D. , Coggill, P. , Eberhardt, R. Y. , Eddy, S. R. , Mistry, J. , Mitchell, A. L. et al. 2016. The Pfam protein families database: towards a more sustainable future. Nucleic Acids Research. 44 (D1): D279-D285. https://doi.org/10.1093/nar/gkv1344

Gal, D., Etti, O., Rinat, O. 2003. Changes in anthocyanin concentration and composition in Jaguar rose flowers due to transient high-temperature conditions. Plant Sci. 164:333-340.

Gantasala, N.P., Papolu, P.K., Thakur, P.K., Kamaraju, D., Sreevathsa, R., Rao, U*. 2013. Selection and validation of reference genes for quantitative gene expression studies by real-time PCR in eggplant (Solanum melongena L). BMC Res Notes. 6: 312. https://doi.org/10.1186/1756-0500-6-312

Harborne, J.B., Williams, C.A. 2000. Advances in flavonoid research since 1992. Phytochemistry. 55:481-504. https:/doi.org/10.1016/S0031-9422(00)00235-1

Hirakawa, H., Shirasawa, K., Miyatake, K., Nunome, T., Negoro, S., Ohyama, A. et al. 2014. Draft genome sequence of eggplant (Solanum melongena L.): the representative solanum species indigenous to the old world. DNA Res. 21 (6):649-660. https://doi.org/ 10.1093/ dnares/ dsu027

Ilk, N., Ding, J., Ihnatowicz, A., Koornneef, M. \& Reymond, M. 2015. Natural variation for anthocyanin accumulation under high-light and low-temperature stress is attributable to the enhancer of ag-4 2 (HUA2) 

locus in combination with production of anthocyanin pigment1 (PAP1) and PAP2. New Phytol. 206(1): $422-435$.

Jiang, M., Liu, Y., Ren, L., Lian, H., Chen, H. 2016. Molecular cloning and characterization of anthocyanin biosynthesis genes in eggplant (Solanum melongena L.). Acta Physiol. Plant. 38:163. https://doi.org/ $10.1007 / \mathrm{s} 11738-016-2172-0$

in, J, Tian, F., Yang, D.C., Meng, Y.Q., Kong, L., Luo, J.C., Gao, G. 2017. Plant TFDB 4.0: toward a central hub for transcription factors and regulatory interactions in plants. Nucleic Acids Res. 45 (D1): D1040-D1045. https://doi.org/10.1093/nar/gkw982

Kim, D., Langmead, B., Salzberg, S.L. 2015. HISAT: a fast spliced aligner with low memory requirements. Nat Methods. 12 (4): 357-360. doi:10.1038/nmeth.3317

Kolde, R. 2012. pheatmap: Pretty Heatmaps. R package version 061.

Li, J., He, Y.J., Zhou, L.; Liu, Y., Jiang, M.M., Ren, L., Chen, H.Y. 2018. Transcriptome profiling of genes related to light-induced anthocyanin biosynthesis in eggplant (Solanum melongena L.) before purple color becomes evident. BMC Genomics. 19:201. https://doi.org/10.1186/ s12864-018-4587-z

Lin J.S., Lin C.C., Lin H.H., Chen Y.C., Jeng S.T. 2012. MicroR828 regulates lignin and $\mathrm{H}_{2} \mathrm{O}_{2}$ accumulation in sweet potato on wounding. New Phytologist. 196:427-440. doi:10.1111/j.1469-8137.2012.04277.x

Lin-Wang, K., Micheletti, D., Palmer, R., Volz, J., Lozano, L., Espley, R. et al. 2011. High temperature reduces apple fruit colour via modulation of the anthocyanin regulatory complex. Plant Cell \& Environ. 34:1176-1190. doi:10.1111/j.1365-3040. 2011.02316.x

Livak, K.J. and Schmittgen, T.D. 2001. Analysis of relative gene expression data using Real-Time quantitative PCR and the 2(-Delta Delta C(T)) method. Methods. 25 (4): 402-408. doi: 10.1006/ meth. 2001.1262

Love, M.I., Huber, W., Anders, S. 2014. Moderated estimation of fold change and dispersion for RNA-seq data with DESeq2. Genome Biol. 15(12):550. http://dx.doi.org/10.1186/s13059- 014-0550-8

Mellway R. D., Tran L. T., Prouse M. B., Campbell M. M. and Constabel C. P. 2009. The wound-, pathogen-, and ultraviolet B-responsive MYB134 gene encodes an R2R3 MYB transcription factor that regulates proanthocyanidin synthesis in poplar. Plnat Physiology. 150: 924-941.

Mori, K., Goto-Yamamoto, N., Kitayama, M., Hashizume, K. 2007. Loss of anthocyanins in red-wine grape under high temperature. J. Exp. Bot. 58:1935-1945. doi:10.1093/jxb/erm055 
Pavel, Z. , Elena, A. , Anna, L. , Natalia, T. , \& Anatoly, V. 2018. Anthocyanin composition and content in rye plants with different grain color. Molecules. 23(4), 948. doi: 10.3390/molecules23040948

Poudel, P.R., Mochioka1, R., Beppu, K. and Kataoka, I. 2009. Influence of temperature on berry composition of interspecific hybrid wine grape 'Kadainou R-1' (Vitis ficifolia var. ganebu $\times$ V. vinifera 'Muscat of Alexandria'). J Japan Soc Hort Sci. 78:169-174.

Rahim, M.A., Busatto, N. \& Trainotti, L. 2014. Regulation of anthocyanin biosynthesis in peach fruits. Planta. 240: 913. https://doi.org/10.1007/s00425-014-2078-2

Rowen, D.D., Cao, M.S., Lin-Wang, K., Cooney, J.M., Jensen, D.J., Austin, P.T. et al. 2009. Environmental regulation of leaf colour in red 35S:PAP1 Arabidopsis thaliana. New Phytol. 82:102-115.

Schulz, E., Tohge, T., Zuther, E., Fernie, A.R., Hincha,* D.K.2016. Flavonoids are determinants of freezing tolerance and cold acclimation in Arabidopsis thaliana. Sci. Rep. 34027.

Shao, W.T. 2013. Cloning and function analysis of anthocyanin-related transcription factor gene $\operatorname{SmMYB}$ in eggplant (Solanum melongena). Master thesis, Shanghai Jiao Tong University, Shanghai. pp:16.

Stommel, J.R. \& Dumm, J.M. 2015. Coordinated regulation of biosynthetic and regulatory genes coincides with anthocyanin accumulation in developing eggplant fruit. J. Am. Soc. Hortic. Sci. 140 (2):129-135.

Takeda, K., Tochika, Y., Fukazawa, R., \& Mori, T. 1994. Flavonoids as uv-protectant. Acta Hortic. 83 (381): $348-354$.

Tang, Q.Y., Feng, M.G. 2007. DPS data processing system: Experimental disign, statistical analysis and data mining. Beijing: Science Press.

Tatusov, R.L., Fedorova, N.D., Jackson, J.D., Jacobs, A.R., Kiryutin, B., Koonin, E.V. et al. 2003. The COG database: an updated version includes eukaryotes. BMC Bioinformatics. 4:41. doi: 10.1186/1471-2105-4-41

Trapnell, C, Williams, B.A., Pertea, G., Mortazavi, A., Kwan, G., van Baren, M.J. et al. 2010. Transcript assembly and quantification by RNA Seq reveals unannotated transcripts and isoform switching during cell differentiation. Nature Biotechnology. 28(5):511-515.

Treutter, D. 2005. Significance of flavonoids in plant resistance and enhancement of their biosynthesis. Plant Biol. 7: 581-591. doi:10.1055/s-2005-873009

Wang, F., Zhu, H., Chen, D., Li, Z., Peng, R., Yao, Q. 2016. A grape bHLH transcription factor gene, VvbHLH1, increases the accumulation of flavonoids and enhances salt and drought tolerance in transgenic arabidopsis thaliana. Plant Cell Tiss. Org. 125 (2): 387-398. doi: https://doi.org /10.1007/ s11240-016-0953-1 
Wang, S.J. 2016. Screening and cloning of key MYB transcription factor of anthocyanin biosynthesis in eggplant (Solanum melongena L.). Master thesis, Shandong Agricultural University, Taian.

Winkel-Shirley B.2001. Flavonoid biosynthesis: a colorful model for genetics, biochemistry, cell biology, and biotechnology. Plant Physiol. 126(2): 485-493.

Ye, J., Fang, L., Zheng, H., Zhang, Y., Chen, J., Zhang, Z. et al. 2006. WEGO: a web tool for plotting GO annotations. Nucleic Acids Res. 34 (2): W293-297. doi:10.1093/nar/gk1031

Ye, J., Coulouris, G., Zaretskaya, I., Cutcutache, I., Rozen, S., Madden, T.L. 2012. Primer-BLAST: A tool to design target-specific primers for polymerase chain reaction [J]. BMC bioinformatics. 13:134-144. PMID: 22708584

Zhai, R., Wang, Z., Zhang, S., Meng, G., Song, L., Wang, Z. et al. 2016. Two MYB transcription factors regulate flavonoid biosynthesis in pear fruit (pyrus bretschneideri rehd.). J. Exp. Bot. 67:1275-1284. doi: 10.1093/jxb/ erv524

Zhang, Y.J., Hu, Z.L., Chu, G.H., Huang, C., Tian, S.B., Zhao, Z.P. and Chen, G.P. 2014. Anthocyanin accumulation and molecular analysis of anthocyanin biosynthesis-associated genes in eggplant (Solanum melongena L.). J. Agric. Food Chem. 2(13): 2906-2912. doi:10.1021/jf404574c

Zhou, H., Lin-Wang, K., Wang, H., Gu, C., Dare, A.P., Espley, R.V. et al. 2015. Molecular genetics of blood-fleshed peach reveals activation of anthocyanin biosynthesis by NAC transcription factors. Plant J. Cell \& Mol. Bio. 82(1): 105. doi:10.1111/ tpj.12792 


\section{Captions}

Fig. 1 (A): Eggplant E30 with normal purplish-red peel; (B): Eggplant E30 with poor peel color under high temperature; (C): The content of total anthocyanin in eggplant peels between $35{ }^{\circ} \mathrm{C}$ and $25{ }^{\circ} \mathrm{C}$ treatments. 10DAF: on the $10^{\text {th }}$ day after flowering; 15DAF: on the $15^{\text {th }}$ day after flowering; 20DAF: on the $20^{\text {th }}$ day after flowering. HT: $35^{\circ} \mathrm{Chigh}$ temperature treatment; Control: $25^{\circ} \mathrm{C}$ treatment. Values are the means $\pm \mathrm{SD}$ of three biological replicates. Asterisks indicate a significant difference determined by the Duncan test $(\mathrm{p}<0.01)$

Fig. 2 Differentially expressed genes of E30 (Solanum melongena) between $35{ }^{\circ} \mathrm{C}$ and $25{ }^{\circ} \mathrm{C}$ treatments. 10DAF_up, 15DAF_up, 20DAF_up: the up-regulated genes on the $10^{\text {th }}, 15^{\text {th }}$ and $20^{\text {th }}$ days after flowering respectively. 10DAF_down, $15 \mathrm{DAF} \_$down, 20DAF_down: the down-regulated genes on the $10^{\text {th }}, 15^{\text {th }}$ and $20^{\text {th }}$ days after flowering respectively.

Fig. 3 KEGG Pathway Classification of differentially expressed genes in Solanum melongena between $35{ }^{\circ} \mathrm{C}$ and $25{ }^{\circ} \mathrm{C}$ treatments. (A): on the $10^{\text {th }}$ day after flowering; (B): on the $15^{\text {th }}$ day after flowering; $(\mathbf{C})$ : on the $20^{\text {th }}$ day after flowering.

Fig. 4 Heat map depicting log2 (Fold Change) of differentially expressed genes in Flavonoid biosynthetic category of Solanum melongena between $35{ }^{\circ} \mathrm{C}$ and $25{ }^{\circ} \mathrm{C}$ treatments.

Fig. 5 qRT-PCR validation of RNA-Seq data using 12 peel-coloring related genes in Solanum melongena. $18 S$ $r R N A$ expression values were used as the internal reference. Values are the means \pm SD of three biological replicates. 10DAF: on the 10th day after flowering; 15DAF: on the 15th day after flowering; 20DAF: on the 20th day after flowering.

Fig. 6 The KEGG pathway enrichment scatter plot of differentially expressed transcription factors in Solanum melongena between $35{ }^{\circ} \mathrm{C}$ and $25{ }^{\circ} \mathrm{C}$ treatments. (A): on the $10^{\text {th }}$ day after flowering; (B): on the $15^{\text {th }}$ day after flowering; (C): on the $20^{\text {th }}$ day after flowering. 
446 Fig. 7 Biosynthetic pathways of anthocyanin, flavonol and lignin (Mellway et al. 2009)

447 ANS, Anthocyanidin synthase; CCoAOMT, Caffeoyl-CoA O-methyltransferase; CHI, Chalcone isomerase; CHS, 448 Chalcone synthase; CYP, Cytochrome P450; C3H, Coumaric acid-3-hydroxylase; C4H, Cinnamate -4-hydroxylase; DFR, 449 Dihydroflavonol reductase; F3'5'H, Flavonoid 3',5'-hydroxylase; F3'H, Flavonoid 3'-hydroxylase; F3H, Flavanone 3-hydroxylase; FLS, flavonol synthase; PAL, Phenylalanine ammonialyase

451 

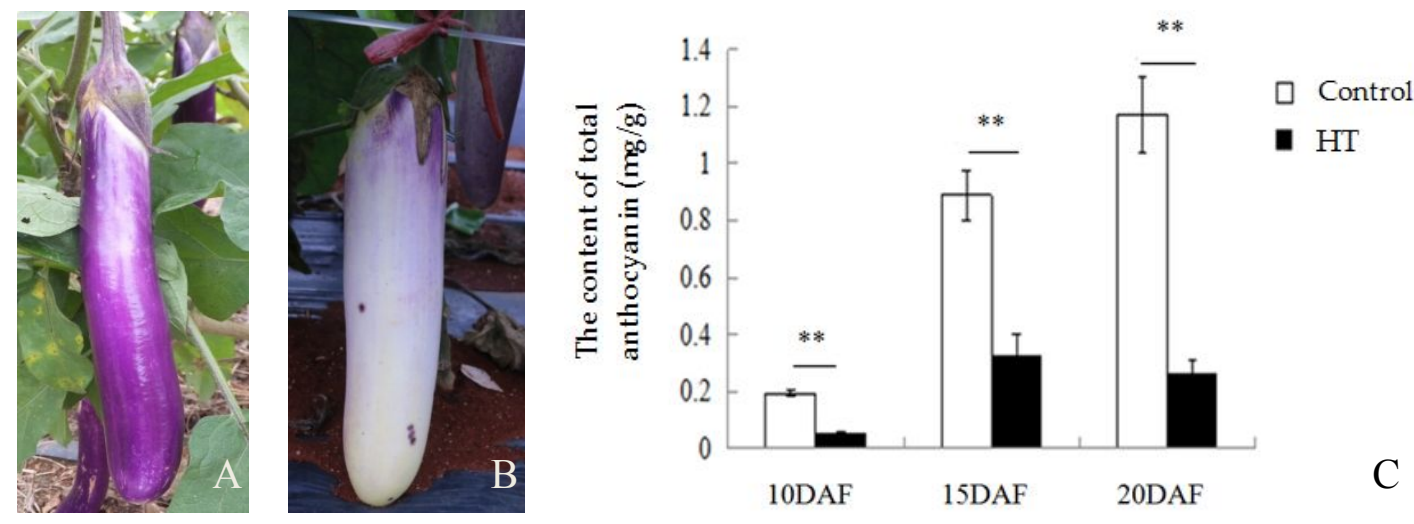


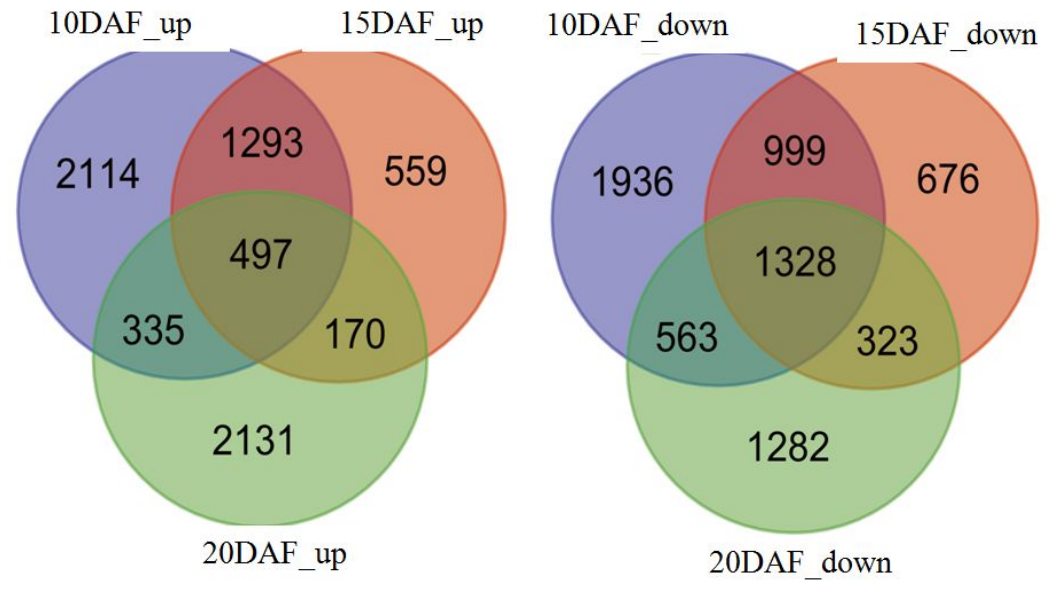


A $\quad$ Different expression gene $\square$ Corrected p-value

\begin{tabular}{l|l|l|l|} 
Glyoxylate and dicarboxylate.. 21 & $1.77 \mathrm{E}-02$
\end{tabular}

Plant hormone signal transduction

86

6.43E-03

alpha-Linolenic acid metabolism

$13 \quad 2.25 \mathrm{E}-03$

\begin{tabular}{|l|l|l|}
\hline Flavonoid biosynthesis & 20 & $2.62 \mathrm{E}-06$ \\
\hline
\end{tabular}

Biosynthesis of unsaturated fatty.. $26 \quad 2.61 \mathrm{E}-06$

Plant-pathogen interaction

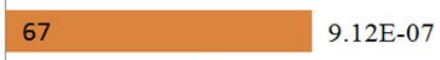

Phenylalanine metabolism

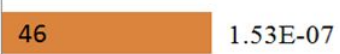

Phenylpropanoid biosynthesis

47

$9.86 \mathrm{E}-09$

$\begin{array}{llllll}0 & 20 & 40 & 60 & 80 & 100\end{array}$

B.

$\varpi$ Different expression gene $₫$ Corrected p-value

\begin{tabular}{l|l|l} 
Phenylalanine metabolism & 26 & $1.68 \mathrm{E}-02$
\end{tabular}

\begin{tabular}{lll}
\hline Flavonoid biosynthesis & 12 & $1.44 \mathrm{E}-02$
\end{tabular}

Biosynthesis of unsaturated fatty.. $16 \quad 7.46 \mathrm{E}-03$

Plant hormone signal transduction

Phenylpropanoid biosynthesis

61

4.29E-03

Protein processing in.

$29 \quad 3.59 \mathrm{E}-04$

$54 \quad 1.62 \mathrm{E}-04$

Plant-pathogen interaction

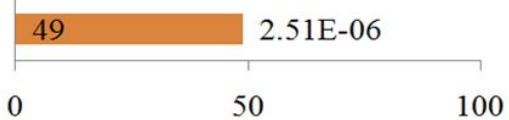

C Phenylalanine metabolism $\quad 32 \quad 2.48 \mathrm{E}-02$

\begin{tabular}{|c|c|}
\hline Flavonoid biosynthesis & $14 \quad 2.11 \mathrm{E}-02$ \\
\hline Biosynthesis of unsaturated fatty.. & $1.06 \mathrm{E}-02$ \\
\hline DNA replication & $9.00 \mathrm{E}-03$ \\
\hline Pentose phosphate pathway & 8.89E-03 \\
\hline Carotenoid biosynthesis & $8.55 \mathrm{E}-04$ \\
\hline Phenylpropanoid biosynthesis & 7.24E-05 \\
\hline Carbon fixation in photosynthetic.. & $1.54 \mathrm{E}-06$ \\
\hline Photosynthesis - antenna proteins & $2.17 \mathrm{E}-11$ \\
\hline Photosynthesis & $6.06 \mathrm{E}-13$ \\
\hline Porphyrin and chlorophyll.. & $4.96 \mathrm{E}-14$ \\
\hline
\end{tabular}

$\begin{array}{llllllll}0 & 10 & 20 & 30 & 40 & 50 & 60 & 70\end{array}$

$\llbracket$ Different expression gene $₫$ Corrected $p$-value 


\begin{tabular}{|c|c|c|c|}
\hline $\begin{array}{l}10 \text { days after } \\
\text { flowering }\end{array}$ & $\begin{array}{c}15 \text { days after } \\
\text { flowering }\end{array}$ & $\begin{array}{c}20 \text { days after } \\
\text { flowering }\end{array}$ & \\
\hline-1.04 & 0.00 & 0.00 & Sme2.5_00015.1_g00020.1_F3H \\
\hline-4.29 & -1.23 & -3.10 & Sme2.5_00029.1_g00005.1_Caffeoyl-CoA O-methyltransferase (CCOAMT-2) \\
\hline-4.65 & -1.14 & -2.31 & Sme2.5_00776.1_g00002.1_Caffeoyl-CoA 0-methyltransferase (CCOAMT-1) \\
\hline-1.06 & 0.00 & 0.00 & Sme2.5_00065.1_g00021.1_flavonoid 3\&apos; ;\&kapos;-methyltransferase \\
\hline-13.20 & -2.28 & -3.59 & Sme2.5_00085.1_g00033.1_Cytochrome P450 98A3 (CYP 98A3-1) \\
\hline-2.44 & -2.44 & -3.58 & Sme2.5_00085.1_g00034.1_Cytochrome P450 98A2 (CYP 98A2-1) \\
\hline-12.11 & -2.41 & -3.56 & Sme2.5_00085.1_g00035.1_CytochromeP450(CYP) \\
\hline 1.40 & 1.42 & 0.00 & Sme2.5_00283.1_g00002.1_CHS \\
\hline-2.10 & 0.00 & -1.67 & Sme2.5_00410.1_g00007.1_Cytochrome P450 CYP73A100-like (CYP73A100) \\
\hline-3.29 & 0.00 & 2.55 & Sme2.5_01077.1_g00016.1_CHS \\
\hline-1.75 & 0.00 & 0.00 & Sme2.5_01193.1_g00009.1_CHI \\
\hline-2.31 & -1.48 & 1.44 & Sme2.5_01401.1_g00004.1_DFR2 \\
\hline-1.57 & -1.07 & 1.03 & Sme2.5_01638.1_g00003.1_ANS2 \\
\hline-1.57 & -1.07 & 1.03 & Sme2.5_01638.1_g00005.1_ANS \\
\hline-1.78 & 0.00 & 1.16 & Sme2.5_01772.1_g00002.1_F3'H \\
\hline-1.63 & -1.21 & 0.00 & Sme2.5_04313.1_g00001.1_E3'5'H \\
\hline-2.85 & 0.00 & 3.01 & Sme2.5_13923.1_g00001.1_CHS \\
\hline-7.70 & -3.35 & -5.17 & Sme2.5_29908.1_g00001.1_CCOAMT-3 \\
\hline-6.38 & 0.00 & 0.00 & XLOC 002669 CYP 98A3-2 \\
\hline-1.38 & -1.38 & 0.00 & XLOC_046563_F $3^{\prime} 5^{\prime} \mathrm{H}$ \\
\hline 0.00 & -1.23 & 0.00 & Sme2.5_00529.1_g00005.1_CytochromeP450 98A2-like（CYP 98A2-2) \\
\hline 0.00 & 0.00 & 2.34 & Sme2.5_00662.1_g00008.1_Shikimate 0-hydroxycinnamoyltransferase-like \\
\hline
\end{tabular}



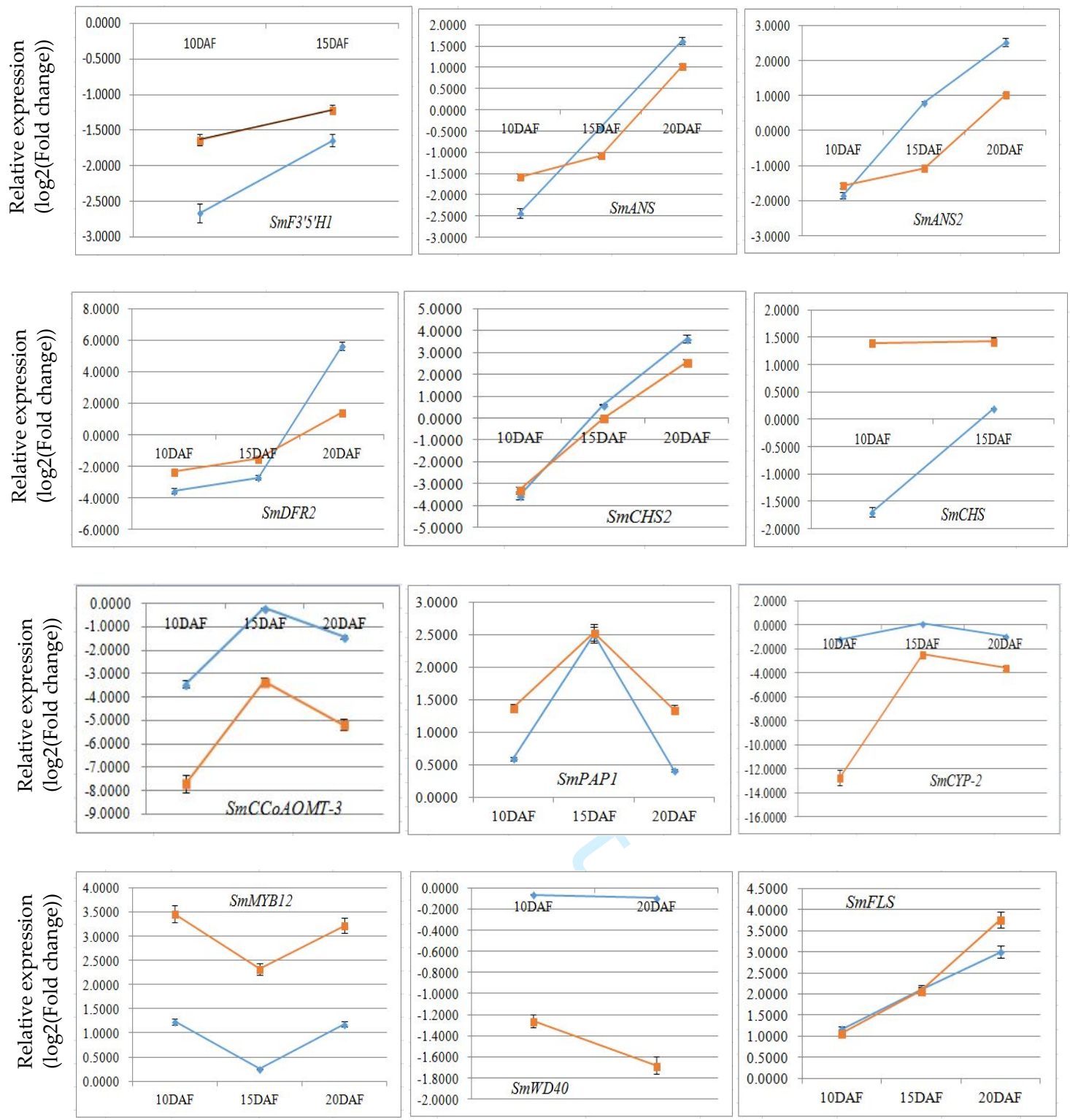

qRT-PCR

RNA-seq 


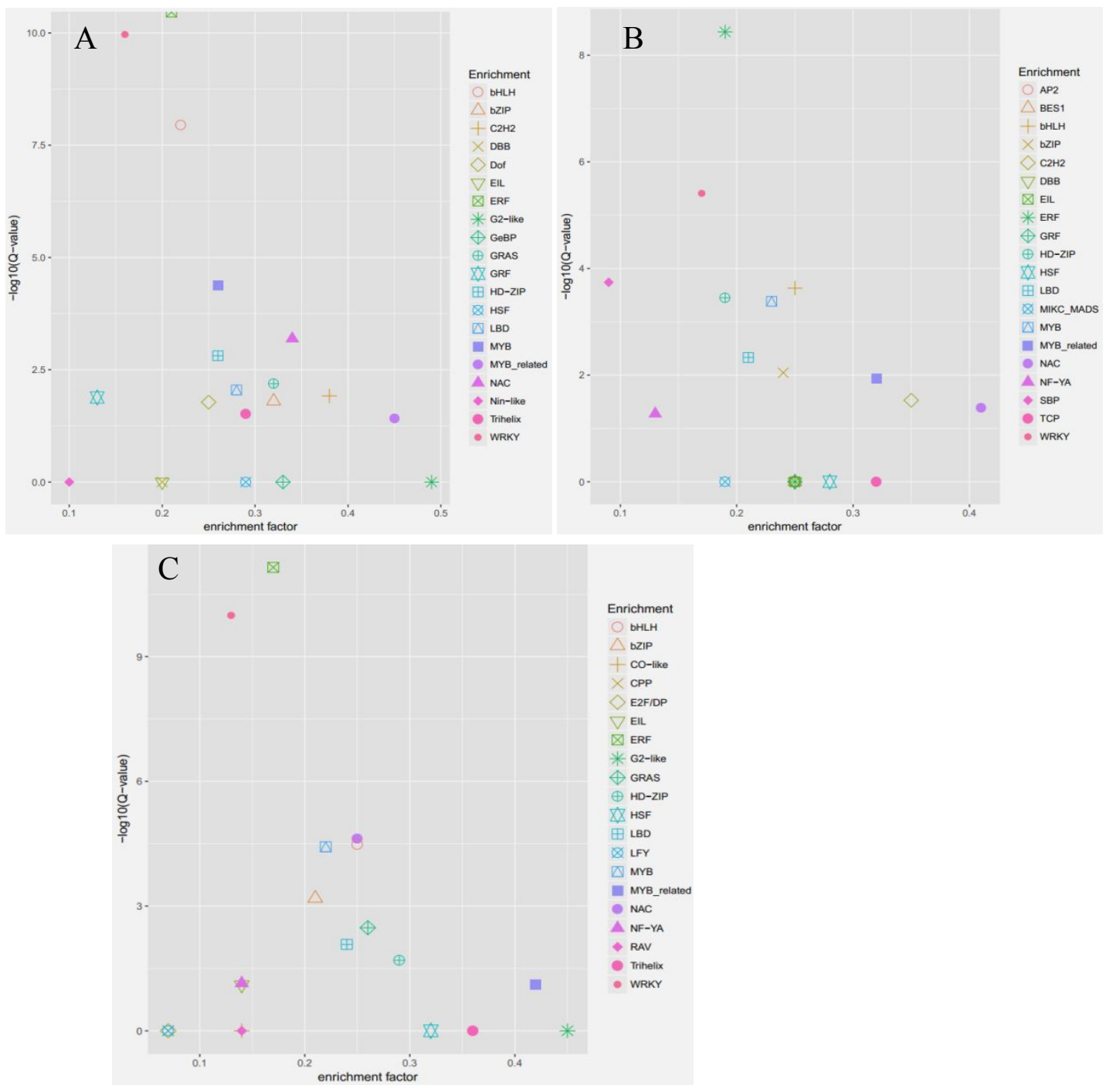




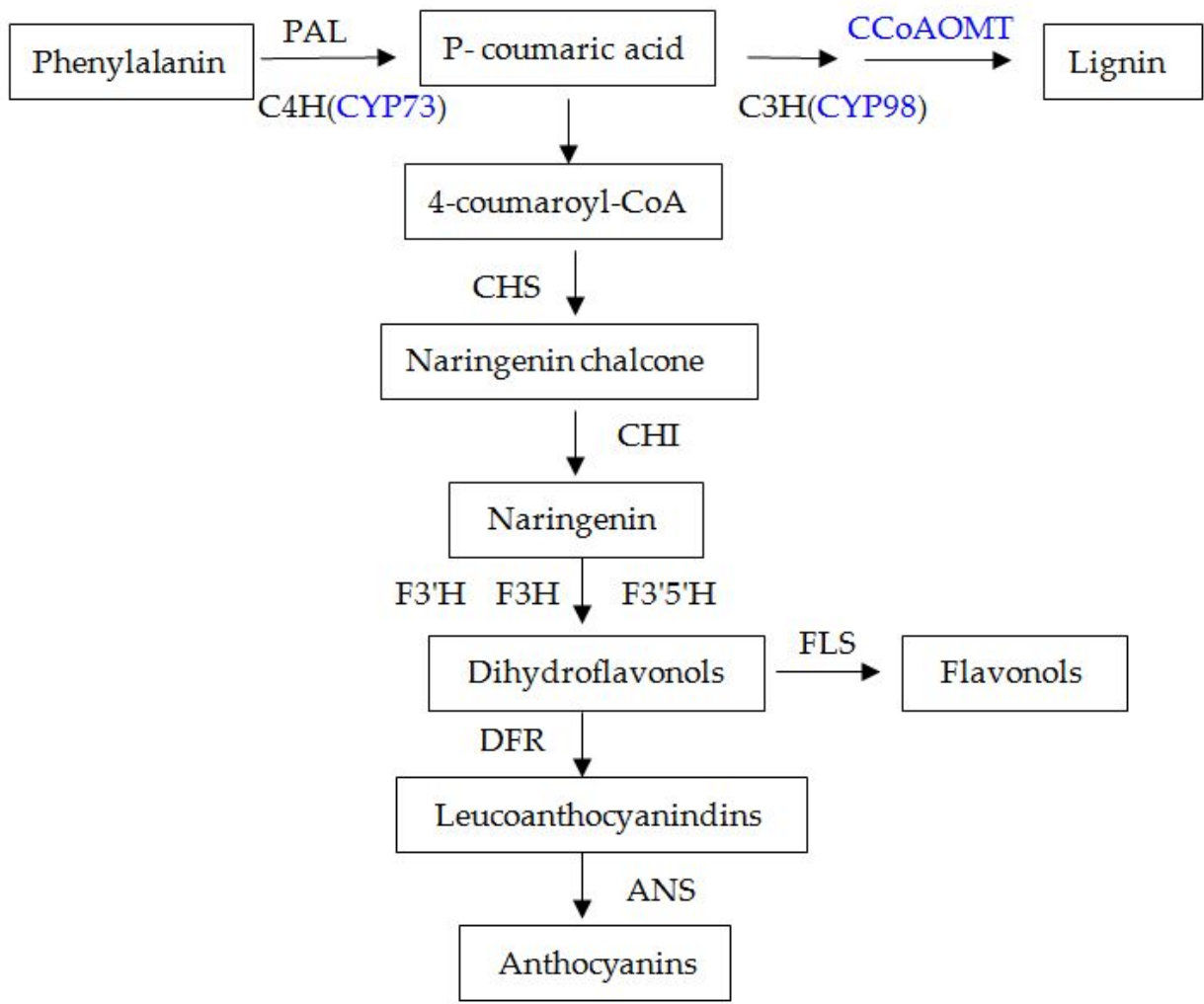

